\title{
Datenbasierte Verhaltensanalyse mit Fuzzy Graphen
}

\author{
Klaus-Peter Huber und Michael R. Berthold \\ Universität Karlsruhe, IRF (Prof. Dr. D. Schmid), Zirkel 2, 76128 Karlsruhe \\ Email:kphuber@informatik.uni-karlsruhe.de, berthold@informatik.uni-karlsruhe.de
}

\begin{abstract}
Zusammenfassung. Im Umfeld immer komplexer werdender technischer Systeme wird die Analyse des Systemverhaltens immer wichtiger. In vielen Anwendungen werden dazu Modelle generiert und ausgewertet. Aus den gewonnenen Daten können Erkenntnisse über das Verhalten extrahiert werden. Eine Möglichkeit besteht darin, ein dem Modell verhaltensähnliches Metamodell zu generieren. In diesem Beitrag wird dazu ein Ansatz vorgestellt, der aus Daten automatisch einen Fuzzy Graphen generiert, der eine kompakte Darstellung des zu approximierenden Verhaltens erlaubt und in verständlichen Wenn-Dann-Regeln dargestellt werden kann. Mit den erzeugten Regeln können Abhängigkeiten zwischen Modellparametern erkannt werden, und das Fuzzy Graph Metamodell kann zum Beispiel zur Einsparung zeit- und kostenintensiver Simulationsexperimente genutzt werden. An einem komplexen Modell eines Feldbus-Systems wird die Anwendung von Fuzzy Graph Metamodellen aufgezeigt.
\end{abstract}

Schlüsselwörter. Funktionsapproximation, Regeln, Fuzzy Regeln, Fuzzy Graph, Modell, Simulation, Metamodell, Verhaltensanalyse

\section{Motivation}

In vielen technischen Anwendungen erfolgt heute die Systemanalyse mit Modellen, durch die bestimmte Eigenschaften des zu modellierenden Systems nachgebildet werden können. Durch Abstraktion und Idealisierung wird eine Komplexitätsreduktion erreicht, und das Modell kann ausgewertet werden, ohne den Betrieb eines vorhandenen Systems zu stören oder zu gefährden, bzw. ohne die Entwicklung teurer Prototypen notwendig zu machen. Aus Sicht des Modellierers interessieren Änderungen bestimmter am System beobachtbarer Zielgrößen in Abhängigkeit von einzelnen Parametern. Diese Fragestellung wird im folgenden als Verhaltensanalyse eines Modells verstanden. Die Verhaltensanalyse kann verschiedene Ziele haben. Bei der Parameteroptimierung geht es darum, eine Einstellung der Modelleingabeparameter zu finden, so da $\beta$ eine vorzugebende 
Zielfunktion optimal wird. Dazu werden verschiedene Suchverfahren eingesetzt und kombiniert (Syrjakow 1997). Eine weitergehende Fragestellung ist die Empfindlichkeitsanalyse. Hier ist das Ziel, den Einfluß einzelner Parameter kennenzulernen (Kreimer 1992). Eine andere wichtige Aufgabe besteht darin, das implizit im Modell enthaltene Wissen über Abläufe und Abhängigkeiten dem Modellierer explizit zu machen, um damit Schwächen, Fehler und das Gesamtverhalten besser verstehen zu können (Pierreval 1992).

Wir betrachten im folgenden Modelle, deren Verhalten sich im Sinne einer Funktion, die Eingabeparameter auf Zielgrößen abbildet, beschreiben läßt:

$$
f_{\text {Modell }}\left(x_{1}, \ldots, x_{m}\right)=\left(y_{1}, \ldots, y_{o}\right)
$$

Da keine Informationen über den internen Modellaufbau einfliessen, wird das Verhalten des Modells als Blackbox-Funktion, die Eingabeparameter auf Zielgrößen abbildet, interpretiert (Abb. 1).

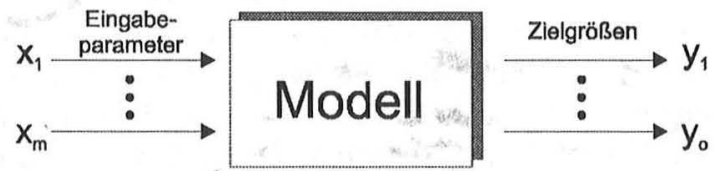

Abb. 1: Das Modellverhalten als Blackbox-Funktion

Für einen gegebenen Satz von $m$ Eingabeparameterwerten $\left(\mathrm{x}_{1} . . \mathrm{x}_{\mathrm{m}}\right)$ wird für $o$ verschiedene Ausgabeparameter je ein Wert durch Auswertung des Modells bestimmt. Da gerade im technischen Bereich viele Modelle auf Basis stochastischer Simulationen ausgewertet werden, sind die Zielgrößenwerte nicht immer eindeutig, sondern können einem "Rauschen" unterliegen.

Zur Verhaltensanalyse von Modellen gibt es strukturbasierte Ansätze, die sich jedoch nur für nicht zu komplexe Modelle mit ganz speziellen Strukturen eignen. In der Praxis werden daher oft datenbasierte Ansätze verwendet, die eine Analyse allein mit Daten von Modellauswertungen ermöglichen. Hierbei bilden die Metamodellierungsansätze den Schwerpunkt. Ziel ist es, aus den Daten ein Modell des Modells (Metamodell) zu erzeugen, so daß dieses Modell ein vergleichbares Verhalten zeigt (Abb. 2). Vergleichbares Verhalten heißt, daß die vom Metamodell approximierte Funktion der Funktion des Modells entspricht.

Klassische Ansätze zur Metamodellierung nutzen die entsprechenden Daten von Modellauswertungen, um eine funktionale Abbildung zu definieren. Dabei werden meist Regressionsgleichungen so parametrisiert, daß eine gewisse Approximationsgenauigkeit erreicht wird, siehe Kleijnen (1979). Damit ist das erzeugte Metamodell zwar sehr kompakt, aber die Ausdrucksfähigkeit ist oftmals nicht ausreichend, zumal aus Vereinfachungsgründen oft lineare Zusammenhänge angenommen werden. 


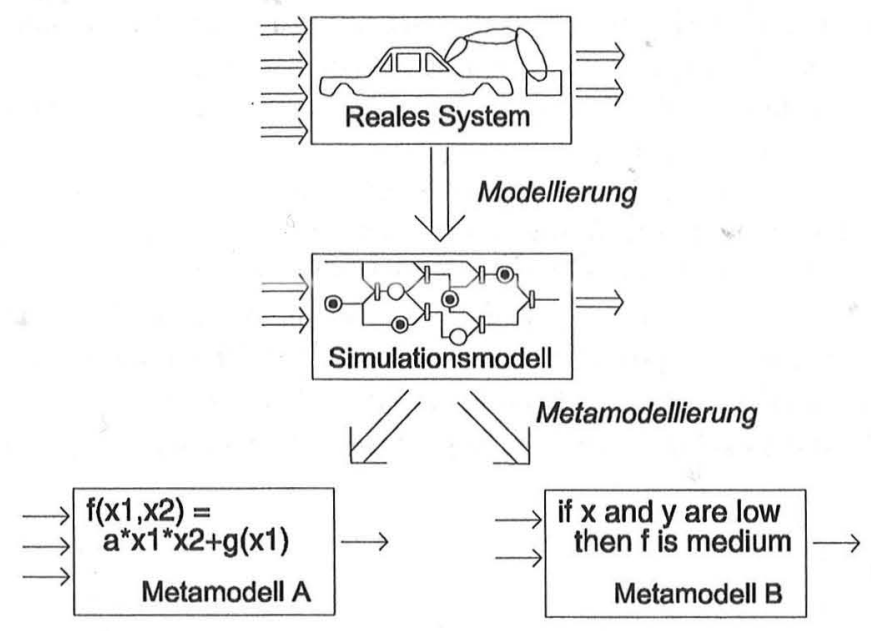

Abb. 2: Metamodelle approximieren das Verhalten eines Modells

Neuere Ansätze setzen auch künstliche neuronale Netze als Metamodelle ein, siehe (Hurrion 1992) oder (Pierreval 1996), wobei hier die Interpretierung noch schwieriger wird. Ansätze mit symbolischen Lernverfahren aus dem Bereich des Maschinellen Lernens liefern zwar verständliche regelbasierte Metamodelle, stellen aber nur ein sehr grobes Metamodell dar (Pierreval 1992).

Der hier vorgestellte Lösungsansatz setzt einen Algorithmus ein, der WennDann-Regeln aus Modelldaten generiert. Basierend auf einem sehr effizienten Lernverfahren werden automatisch Regeln erzeugt, wobei die Regeln intern als Fuzzy Graph repräsentiert sind. Durch eine entsprechende Auswertung ist es möglich, die Regelbasis zur Funktionsapproximation einzusetzen. Das Konzept des Fuzzy Graphen erlaubt es, auch verrauschte Zielgrößenwerte zu verarbeiten. Durch die sehr einfache Interpretation der Regeln wird das Metamodell verständlich und das Verhalten transparent. Da die interne Struktur des eigentlichen Modells nicht berücksichtigt wird, kann dieser Ansatz auf praktisch jedes Modell angewendet werden, dessen Verhalten sich durch Modelldaten beschreiben läßt.

\section{Generierung von Fuzzy Graphen}

Bei der Metamodellierung geht es nun nicht nur darum, die Modellfunktion nachzubilden, vielmehr soll das Metamodell auch einfach zu generieren sein. Zur Funktionsapproximation gibt es in der Fuzzy Logik bereits Methoden, um aus Daten Regeln zu erzeugen. Bei vielen Ansätzen liegt der Schwerpunkt auf einer möglichst genauen Approximation der zugrundeliegenden Daten. Allerdings kann dann die Regelbasis sehr groß (Wang und Mendel 1992) oder die Interpretation der Regeln sehr aufwendig werden (Abe und Lan 1995). Ein anderer unerwünschter Effekt ist bei (Higgins und Goodman 1993) zu beobachten. Dort 
dürfen die Daten nicht zu stark verrauscht sein. Für die Metamodellierung erscheint keiner der beschriebenen Ansätze ausreichend geeignet, insbesondere da die Regelmengen durch eine meist vorgegebene Einteilung der Eingabewertebereiche sehr groß werden.

Bei der Verwendung von Fuzzy Graphen statt Fuzzy Regeln können auch komplexe Funktionen mit wenigen einfachen Elementen (Fuzzy Punkten) beschrieben werden. Der im folgenden vorgestellte Ansatz generiert automatisch einen solchen Fuzzy Graphen anhand von Beispieldaten. Die zentrale Idee des Verfahrens ist, die Zielgrößenwerte durch eine Fuzzifizierung auf überlappende Klassen (Granularisierung) abzubilden, und dann mittels eines effizienten konstruktiven Algorithmus' einen Fuzzy Graphen zu generieren (Abb. 3).

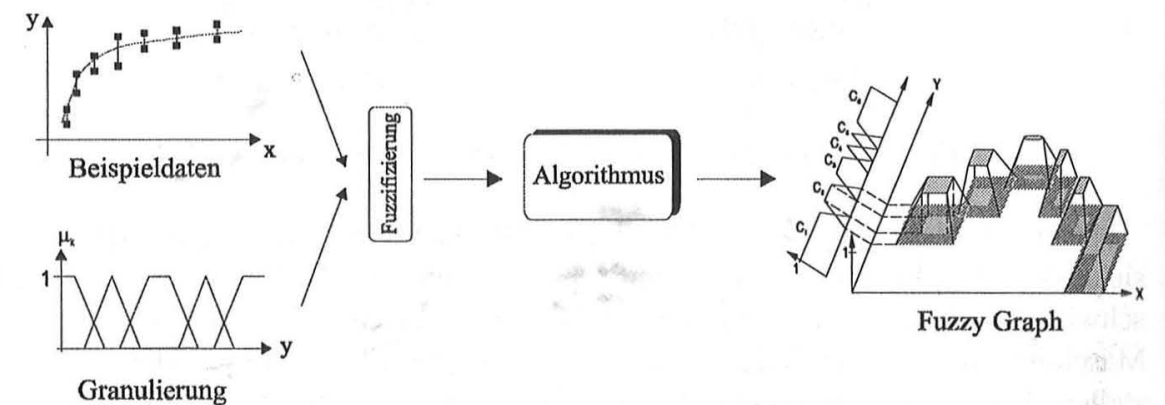

Abb. 3: Erzeugung eines Fuzzy Graphen aus Beispieldaten

\subsection{Fuzzy Graphen}

Der Begriff Fuzzy Graph wurde von Zadeh geprägt und beschreibt einen Graphen, der unscharfe Mengen auf unscharfe Mengen abbildet (Zadeh 1994). Unscharfe Mengen werden durch (hier: endliche trapezförmige) Zugehörigkeitsfunktionen beschrieben, die jedem Element der Grundmenge einen Zugehörigkeitswert zwischen 0 und 1 zuordnen, siehe auch (Bandemer und Gottwald 1993). Eine Fuzzy Funktion beschreibt nun eine Abbildung von unscharfen Mengen eines $m$-dimensionalen Eingabebereiches auf unscharfe Mengen eines eindimensionalen Wertebereiches.

$$
f^{*}: A_{1} \times \ldots \times A_{m} \rightarrow Y
$$

Jedes $A_{i}$ beschreibt eine Zugehörigkeitsfunktion für den Eingabeparameter i. Jeder Punkt des Fuzzy Graphen ist ein sogenannter Fuzzy Punkt $\left(\mathrm{A}_{1}, \ldots, \mathrm{A}_{\mathrm{m}}, \mathrm{Y}\right)$, wobei $A_{i}$ eine Fuzzy Menge des entsprechenden Wertebereiches und $Y$ analog eine Fuzzy Menge des Ausgabewertebereiches darstellt. Ein Fuzzy Graph setzt sich dann aus einer endlichen Menge solcher Fuzzy Punkte zusammen. In Abb. 4 ist ein Beispiel für einen Fuzzy-Graphen für nur einen Eingabeparameter dargestellt. Jeder Fuzzy Punkt überdeckt mit einer eigenen Zugehörigkeitsfunktion A einen Teilbereich des Eingabeparameterraums $X$ und ist direkt assoziiert mit einer 
Zugehörigkeitsfunktion B des Wertebereiches $Y$ des Ausgabeparameters. Damit ist es möglich, eine Funktion $f$ durch wenige Fuzzy Punkte zu beschreiben.

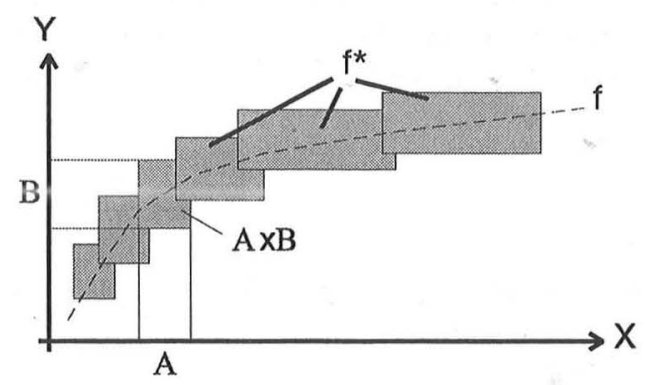

Abb. 4: Fuzzy Graph bei einer Eingabedimension

Der wesentliche Vorteil dieser Repräsentation ist die kompakte und lokale Darstellung der funktionalen Abhängigkeit zwischen dem Eingabebereich und dem Ausgabebereich. Bei einem mehrdimensionalen Eingaberaum ist diese Darstellung analog möglich. Wie später gezeigt wird, kann jeder Fuzzy Punkt eines solchen Fuzzy Graphen als eine Wenn-Dann-Regel dargestellt werden. Bei der Approximation einer Funktion mit einem Fuzzy Graphen wird für einen Punkt $x$ im Eingaberaum die Zugehörigkeit zu jedem Fuzzy Punkt bestimmt, und daraus wird die resultierende Zugehörigkeitsfunktion im Ausgabebereich abgeleitet. Seien $p$ Fuzzy Punkte und der Eingabewert $x$ durch eine Zugehörigkeitsfunktion $\mu_{\mathrm{A}}(\mathrm{x})$ gegeben, dann ergibt die Anwendung des klassischen t-Norm Operators:

$$
\mu(\mathrm{x})=\operatorname{suprenum}_{\mathrm{y}}\left\{\min \left\{\max \left\{\mu_{\mathrm{A} 1 \times \mathrm{B} 1}(\mathrm{x}, \mathrm{y}), \ldots, \mu_{\mathrm{Ap} \times \mathrm{Bp}}(\mathrm{x}, \mathrm{y})\right\}, \mu_{\mathrm{A}}(\mathrm{x})\right\}\right\}
$$

Dieses Vorgehen wird für alle Fuzzy Punkte des Fuzzy Graphen wiederholt. Unter allen Werten der Ausgabegröße wird dann der Fuzzy Punkt mit der maximalen Zugehörigkeit bestimmt. Damit beschreibt der Fuzzy Graph in kompakter und durch die Regeln sehr verständlicher Form den Zusammenhang zwischen unscharfen Mengen. Zur Verarbeitung scharfer Werte können Verfahren angegeben werden, die die scharfen Werte auf unscharfe Werte abbilden und umgekehrt (Bandemer und Gottwald 1993).

\subsection{Automatische Generierung des Fuzzy Graphen}

Zur Generierung des Fuzzy Graphen benötigt der hier vorgestellte Algorithmus eine Granularisierung des Wertebereiches durch $k$ Zugehörigkeitsfunktionen. Diese Zugehörigkeitsfunktionen sollten trapezförmig sein und den Wertebereich der Zielgröße vollständig abdecken. Durch eine Überlappung wird eine "glatte" Approximation kontinuierlicher Zielgrößen erreicht, wobei sich in der Praxis eine paarweise Überlappung als sinnvoll erwiesen hat. Außerdem kann gezeigt werden, daß der Approximationsfehler auf den Beispieldaten durch die Wahl der Granularisierung nach oben beschränkt wird. 
Die einzelnen Zugehörigkeitsfunktionen der Granularisierung können beliebige Breiten haben. Da die Breite die Approximationsgenauigkeit direkt beeinflußt, kann dadurch auf bestimmte Wertebereiche fokussiert werden. Schmale Zugehörigkeitsfunktionen führen zu einer höheren Approximationsgenauigkeit als breitere. In Abb. 5 sind zwei Möglichkeiten skizziert.
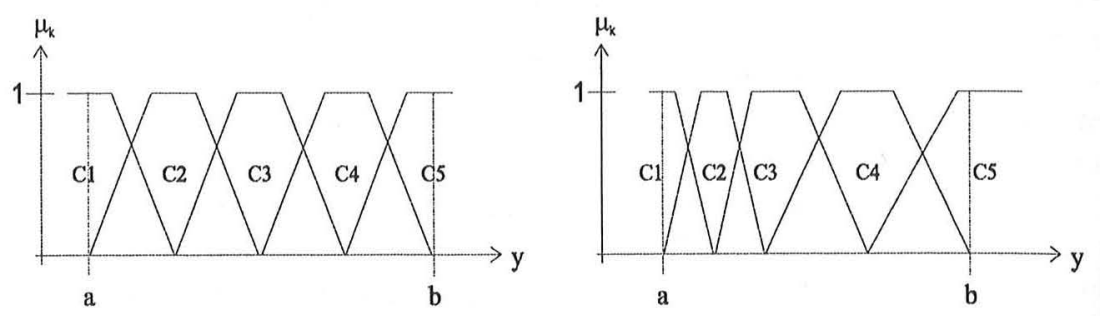

Abb. 5: Granularisierungen können auf einzelne Wertebereiche fokussiert werden

Jeder Zielgrößenwert $y$ eines Beispieldatums kann durch eine trapezförmige Zugehörigkeitsfunktion beschrieben werden. Dadurch können vorhandene Informationen über Unschärfe oder Rauschen direkt eingebracht werden. Beispielsweise wird bei vielen stochastischen Simulationsmodellen neben dem Zielgrößenwert ein Konfidenzintervall angegeben, so daß dessen Breite durch das Trapez repräsentiert werden kann. Jedes Beispieldatum besteht aus einem Eingabevektor $x$ mit $m$ Eingabeparametern und einer Beschreibung des Zielgrößenwertes. Es sei eine Granulierung in $k$ Klassen gegeben. Der Zielgrößenwert kann selbst durch eine Zugehörigkeitsfunktion $\mu_{y}$ (hier: trapezförmig) repräsentiert sein. Trapezförmige Zugehörigkeitsfunktionen sind effizient $\mathrm{zu}$ berechnen und ermöglichen auch die Verwendung scharfer Zielgrößenwerte (Abb. 6).
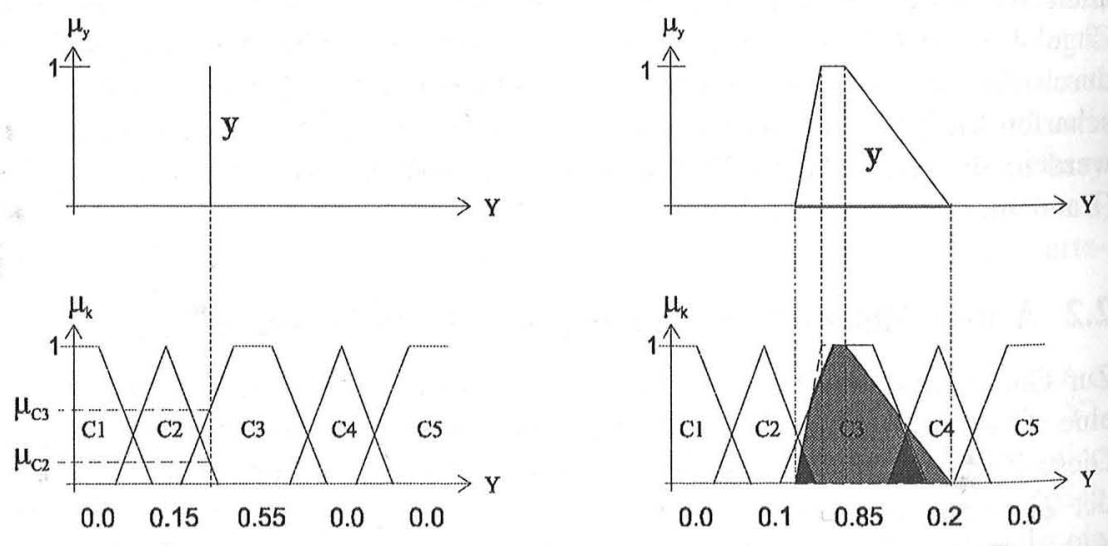

Abb. 6: Fuzzifizierung scharfer und unscharfer Zielgrößenwerte 
Bei der Fuzzifizierung wird der Zielgrößenwert auf Basis der Granularisierung auf einen Zielgrößenvektor $\left(\mu_{1}, \ldots, \mu_{\mathrm{k}}\right)$ abgebildet, indem für jede Klasse die Zugehörigkeit bestimmt wird:

$$
\mu_{c}(y)=\text { suprenum }_{y}\left\{\min \left(\mu_{y}, \mu_{c}\right)\right\},(c=1 \ldots k)
$$

Die Eingabe für den Algorithmus besteht nun aus $n$ Beispieldaten der Form $\left(x_{1}, \ldots, x_{\mathrm{m}}, \mu_{1}, \ldots, \mu_{\mathrm{k}}\right)$. Damit kann die Unschärfe, die in den Zielgrößenwerten der Beispieldaten steckt, explizit über trapezförmige Zugehörigkeitsfunktionen angegeben und bei der Konstruktion des Fuzzy Graphen berücksichtigt werden. Als Ergebnis des Trainings entsteht ein Fuzzy Graph, der eine endliche Anzahl von Fuzzy Punkten enthält. Abbildung 7 zeigt ein Beispiel, wie in dieser Arbeit ein Fuzzy Punkt in einem zweidimensionalen Eingaberaum definiert ist. Der Kern ist der Bereich, in dem die Zugehörigkeit zum Fuzzy Punkt maximal = 1 ist, während im Einflußbereich die Zugehörigkeit vom Kern zur Grenze linear abfällt.

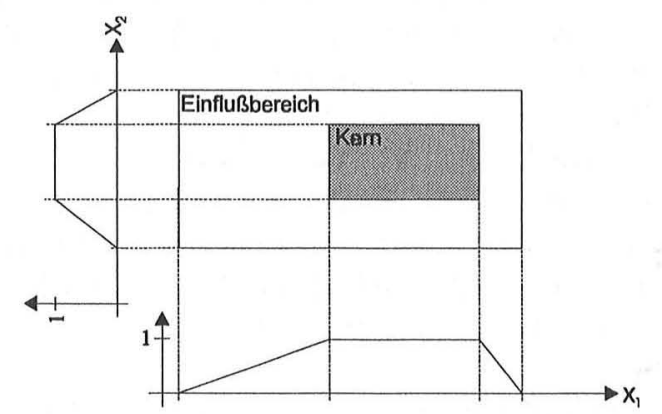

Abb. 7: Ein zweidimensionaler Fuzzy Punkt mit den eindimensionalen Projektionen der Zugehörigkeitsfunktionen

In Abbildung 8 wird gezeigt, wie ein mit dem vorliegenden Verfahren erzeugter Fuzzy Graph den Eingaberaum mit Fuzzy Punkten abdeckt. Jeder Fuzzy Punkt ist einer Klasse zugeordnet und hat einen Kern und einen Einflußbereich.

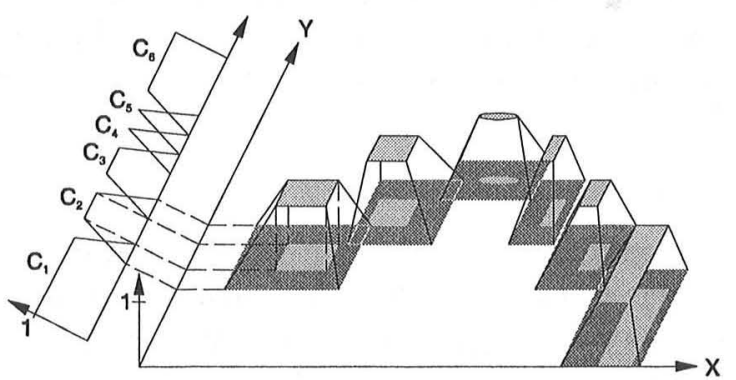

Abb. 8: Ein Fuzzy Graph bei einem eindimensionalen Eingaberaum 
Das Ziel des Algorithmus besteht darin, möglichst große Fuzzy Punkte im $m$ dimensionalen Eingaberaum zu plazieren. Die Grundidee ist, daß alle Beispieldaten von einem Kern der richtigen Klasse überdeckt werden, und daß die Einflußbereiche die Regionen sind, in denen sich kein Beispieldatum „falscher" Klasse befindet. Die ,richtige" Klasse eines Beispieldatums ist diejenige mit maximaler Zugehörigkeit, während alle „falschen“ Klassen die mit Zugehörigkeit 0 sind. Durch die Granularisierung ist das System in der Lage, auch kontinuierliche Funktionen zu approximieren, und der entwickelte Algorithmus erlaubt es, in kurzer Zeit einen leistungsfähigen lokalen Klassifikator zu generieren. Der Trainingsalgorithmus basiert auf einem effizienten Algorithmus, der ursprünglich für die konstruktive Erzeugung neuronaler Netze entwickelt wurde (Huber und Berthold 1995). Er besteht im wesentlichen aus drei Schritten:

- Überdeckung (covered): Liegt ein neues Trainingsmuster x im Einflußbereich eines Fuzzy Punktes, der zur gleichen Klasse k gehört, bei der auch die Zugehörigkeit maximal ist, dann wird der Einflußbereich des Fuzzy Punktes so vergrößert, daß x auch im Kern liegt.

- Einfügung (commit): Gibt es zu dem Trainingsmuster x keinen Fuzzy Punkt der Klasse mit maximaler Zugehörigkeit, dann wird ein neuer Fuzzy Punkt eingefügt. Dieser Fuzzy Punkt beinhaltet als Kern gerade x und der Einflußbereich wird auf unendlich gesetzt.

- Verkleinerung (shrink): Liegt das Trainingsmuster x im Einflußbereich (oder auch im Kern) eines Fuzzy Punktes der Klasse k, wobei der Eingabevektor eine Zugehörigkeit von 0 für diese Klasse hatte, dann wird der Einflußbereich dieses Fuzzy Punktes so verkleinert, daß x gerade herausfällt.

Ein wichtiger Teilschritt betrifft die Verkleinerung der Einflußbereiche bei nicht beabsichtigten Überdeckungen. Mit dem Ziel, möglichst wenige und weit überdeckende Fuzzy Punkte zu erhalten, wird die Verkleinerung so durchgeführt, daß das Volumen des Fuzzy Punktes maximal bleibt. Dazu werden die Werte der Eingabeparameter durch Normalisierung in das Intervall $[0,1]$ abgebildet.

Als Ergebnis des Algorithmus entsteht ein Fuzzy Graph, bei dem die Fuzzy Punkte durch endliche Kerne und durch teilweise bis zum Rand des Wertebereiches reichende Einflußbereiche beschrieben sind. Jedes Beispieldatum liegt dabei in mindestens einem Kern eines Fuzzy Punktes und zwar der Klasse, für die dieses Datum maximale Zugehörigkeit nach der Fuzzifizierung hatte.

\subsection{Approximation mit dem Fuzzy Graphen}

Bei der Funktionsapproximation wird für einen gegebenen Punkt im Eingaberaum $x$ der Wert der Zielgröße bestimmt. Der Fuzzy Graph besteht nun aus einer Menge von Fuzzy Punkten, wobei $\mathrm{p}_{\mathrm{c}}$ Fuzzy Punkte der Klasse $\mathrm{c}=1, \ldots, \mathrm{k}$ zugeordnet sind. Der Eingabevektor $x$ besteht aus $m$ Werten $\left(x_{1}, \ldots, x_{\mathrm{m}}\right)$ der entsprechenden Wertebereiche (hier: Teilmengen der reellen Zahlen). Die Zugehörigkeit des Eingabevektors $x$ zu einem Fuzzy Punkt läßt sich durch den Durchschnitt (nach der 
klassischen t-Norm durch das Minimum repräsentiert) der eindimensionalen Fuzzy Mengen bestimmen. Somit berechnet sich die Zugehörigkeit zum Fuzzy Punkt $i$ der Klasse $\mathrm{c}\left(\mathrm{i}=1, \ldots, \mathrm{m}_{\mathrm{c}}\right)$ :

$$
\mu_{i}^{c}(\vec{x})=\mu_{i}^{c}\left(x_{1}, \ldots, x_{m}\right)=\min \left\{\mu_{i, 1}^{c}\left(x_{1}\right), \ldots, \mu_{i, m}^{c}\left(x_{m}\right)\right\}
$$

Dabei beschreibt $\mu_{i, j}^{c}\left(x_{j}\right)$ die Zugehörigkeit des Punktes in der eindimensionalen Projektion auf den j-ten Wertebereich. Für eine Klasse c wird die Zugehörigkeit entsprechend über alle Fuzzy Punkte dieser Klasse berechnet:

$$
\mu^{c}(\vec{x})=\max _{1 \leq i \leq p_{C}}\left\{\mu_{i}^{c}(\vec{x})\right\}
$$

Diese Berechnung erfolgt analog über alle k Klassen und liefert einen Zugehörigkeitsvektor $\left(\mu_{1}, \ldots, \mu_{\mathrm{k}}\right)$ mit $\left(0 \leq \mu_{\mathrm{c}} \leq 1\right.$; $\left.\mathrm{c}=1, \ldots, \mathrm{k}\right)$ als Ergebnis des Eingabevektors $x$. Aus diesem Zugehörigkeitsvektor kann durch Defuzzifizierung ein scharfer Wert bestimmt werden. Hier wird der approximierte Wert durch eine gewichtete Mittelung über die Schwerpunkte der Klassen berechnet:

$$
\tilde{f}(x)=\tilde{y}=\sum_{c=1}^{k}\left(\mu_{c} \cdot S_{c}\right) / \sum_{c=1}^{k} \mu_{c},
$$

wobei $S_{\mathrm{c}}$ den Schwerpunkt der Klasse c und $\mu_{\mathrm{c}}$ den aus dem Fuzzy Graphen berechneten Zugehörigkeitswert zu der Klasse $\mathrm{c}$ beschreibt. Diese Berechnung ist sehr schnell möglich und bildet eine gute Approximation, insbesondere wenn die Zugehörigkeitsfunktionen triangulär oder trapezförmig sind.

Für das vorliegende Verfahren kann bewiesen werden, daß durch die Wahl der Granularisierung der Approximationsfehler auf den zur Generierung des Fuzzy Graphen benutzten Datenpunkten (Trainingsdaten) explizit nach oben beschränkt werden kann. Werden beispielsweise paarweise überlappende Klassen benutzt, ist die Abweichung nie größer als die halbe Breite der einzelnen Klassen. Das bedeutet, daß die Genauigkeit des Fuzzy Graph Approximators explizit vorgegeben werden kann. Mehr Details dazu finden sich in (Huber 1997).

\subsection{Interpretation des Fuzzy Graphen}

Jeder Fuzzy Punkt des generierten Fuzzy Graphen stellt genau ein Hyperrechteck im m-dimensionalen Eingaberaum dar, wobei die Einflußbereiche auch unbeschränkt sein können:

$$
\left(A_{1} \times A_{2} \times \ldots \times A_{m}, c\right)
$$

$A_{i}$ beschreibt eine trapezförmige Zugehörigkeitsfunktion im Eingaberaum i, und $c$ bezieht sich auf eine der bei der Granularisierung festgelegten Zielgrößenklassen $1 \leq c \leq k$. Damit lassen sich die einzelnen $\mathrm{A}_{\mathrm{i}}$ auch als Bedingung in einer Regelprämisse formulieren und jeder Fuzzy Punkt läßt sich als eine Regel R darstellen:
$\mathrm{R}$ : Wenn $\mathrm{x}_{1}$ in $\mathrm{A}_{1}$ und ... und $\mathrm{x}_{\mathrm{m}}$ in $\mathrm{A}_{\mathrm{m}}$
Dann $\mathrm{y}$ ist $\mathrm{c}$ 
Da sich jede Fuzzy Menge $A_{i}$ in der Eingabedimension i und auch die Klasse $c$ durch einen Kern und einen Einflußbereich beschreiben läßt, ergibt sich R zu:

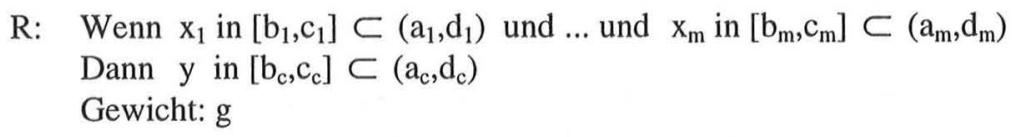

Die Extraktion dieser Regeln aus dem Fuzzy Graph kann sehr schnell erfolgen, da nur die einzelnen Fuzzy Punkte durchlaufen werden müssen und deren Ausdehnung entsprechend ausgegeben wird. Die Parameter $x_{i}$ in den Prämissen entsprechen den Eingabeparametern und y der Zielgröße. Informell kann eine solche Regel folgendermaßen interpretiert werden:

Wenn für einen neuen Punkt alle Werte $x_{i}$ der Eingabeparameter im beschriebenen

Kern [] oder Einflußbereich () dieser Regel liegen, dann liegt der Wert der Zielgröße y in dem durch die Granularisierung vorgegebenen klassenbezogenen Intervall $\left(\left[b_{c}, c_{c}\right] \subseteq\left(a_{c}, d_{c}\right)\right)$. Der Kern dieser Regel wird aufgespannt durch g Trainingsdaten.

Jede Prämisse beschreibt die Bedingungen für einen Parameter durch einen Kern und einen Einflußbereich. Die Beschreibung der Bereiche erfolgt wieder durch ein Trapez mit den äußeren Grenzen $a_{i}$ und $d_{i}$, und den inneren Grenzen $b_{i}, c_{i}$. Bei den Einflußbereichen ist zusätzlich erlaubt, daß die äußeren Grenzen bis zum Rand des Wertebereiches gehen. Wie aus dem oben beschriebenen Algorithmus hervorgeht, beschreibt der Kern einer Regel den kleinsten Bereich, in dem alle Trainingsdaten dieser Regel liegen. Die Grenzen geben genau den minimalen und den maximalen Wert bezogen auf diese Eingabedimension an. Der Einflußbereich ist eine Obermenge des Kerns und beschreibt den Bereich, bis $\mathrm{zu}$ dem kein Trainingsdatum einer „nicht passenden“ Klasse vorhanden ist. Waren keine Daten vorhanden, die zur Einschränkung geführt haben, bleibt der Einflußbereich auf unbeschränkt gesetzt. Für den Zielgrößenwert $y_{i}$ wird die Beschreibung entsprechend der Granularisierung ausgegeben. Das Gewicht einer Regel entspricht der Anzahl an Beispieldaten, die im Kern dieser Regel liegen.

Die Nutzung dieser Informationen wird im folgenden Kapitel beschrieben.

\section{Ergebnisse}

Die beschriebene Generierung eines Fuzzy Graphen aus Beispieldaten kann zur Erzeugung eines Metamodells eingesetzt werden. Um die Modelldaten nutzen zu können, muß zuerst die Granularisierung des Zielgrößenwertebereiches durch die überlappenden Zugehörigkeitsfunktionen (im folgenden Klassen genannt) vorgegeben werden. Liegen Informationen über das Rauschen der Zielgrößenwerte vor, können diese durch individuelle Zugehörigkeitsfunktionen der einzelnen Werte eingebracht werden. Speziell bei stochastischen Simulationsmodellen stellen die Zielgrößenwerte oft Mittelwerte dar, zu denen ein Konfidenzintervall angegeben werden kann. Dieses Konfidenzintervall kann dann auf eine trapezoidale Zugehörigkeitsfunktion abgebildet werden, wie in Abschnitt 2.2 erläutert wurde. Anschließend wird ein Fuzzy Graph erzeugt. Im folgenden wird 
am Beispiel eines komplexen Simulationsmodells gezeigt, wie Fuzzy Graph Metamodelle generiert und analysiert werden können.

Metamodelle sind vor allem dort sinnvoll einsetzbar, wo die realen Systeme und die Simulationsmodelle so komplex sind, daß eine direkte Analyse nur schwer möglich ist. Der vorgestellte Ansatz zur Metamodellierung wurde daher auf ein komplexes Modell aus dem Bereich der Feldbus-Systeme angewandt. FeldbusSysteme werden zur Vernetzung von Maschinen und Rechner im Fertigungsbereich eingesetzt. Wichtige Anforderungen in diesem Umfeld sind die Realzeitfähigkeit, eine hohe Flexibilität und geringe Kosten. In diesem Kapitel wird der Schwerpunkt auf der Analyse der Realzeiteigenschaften liegen, was in unserem Kontext bedeutet, daß die Zeitdauer zur Beantwortung jeder Anfrage unter einer vorgegebenen Schranke liegt. Um diese Eigenschaft zu untersuchen, wurde ein Metamodell in Form eines Fuzzy Graphen generiert und ausgewertet. Schwerpunkt war hier die Untersuchung der Auswirkung einzelner Modellparameter auf die mittleren Antwortzeiten.

\subsection{Modell und Metamodell}

Das modellierte Feldbus-System wurde entsprechend dem ISO/OSI-Standard strukturiert und entwickelt (Abb. 9).

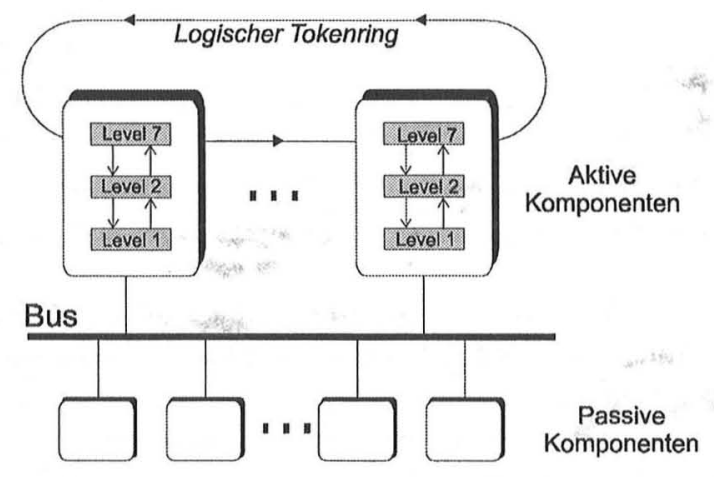

Abb. 9: Architektur des modellierten Feldbus-Systems

Aktive Komponenten können Informationen anfragen oder beantworten, während passive Komponenten nur auf Anfragen reagieren. Jede Station wird durch drei Schichten modelliert, und die Kommunikation wird über einen logischen Tokenring gesteuert. Jede Station kann nur senden und empfangen, wenn sie den Token besitzt. Um dieses Verhalten realistisch nachzubilden, wurde ein komplexes Warteschlangenmodell entwickelt und mit Hilfe einer Simulationsumgebung implementiert und ausgewertet. Die hohe Komplexität mit über 200 Bedienelementen und etwa 20 Eingabeparameter und 10 Zielgrößen macht eine manuelle Analyse der Abhängigkeiten zwischen Modellparametern und Zielgrößen extrem aufwendig und schwierig. 
Zur Untersuchung der Realzeiteigenschaften wird als Zielgröße die mittlere Zeit betrachtet, die bei Anfragen einer aktiven Station bis zur Ankunft der Antwort vergeht. Es wird gefordert, daß diese Antwortzeit unter einer oberen Schranke bleibt, damit das System rechtzeitig auf Ereignisse reagieren kann. Vier Eingabeparameter wurden bezüglich ihrer Einflüsse auf die gewählte Zielgröße berücksichtigt: Mittlere Antwortzeit der CPU, Mittlere Wartezeit zwischen einzelnen Aufträgen, Maximale Target-Rotation-Time und die Anzahl zusätzlicher Stationen. Es wurden 350 Simulationsexperimente (zufällig im Eingaberaum gewählt) durchgeführt und der Wert der mittleren Anwortzeit (rt) bestimmt. Die Werte schwankten dabei zwischen 0.088 und 9.75. Jedes Experiment wurde fünfmal mit jeweils unterschiedlichen Zufallszahlenströmen durchgeführt, um die stochastischen Einflüsse beurteilen zu können. Von den fünf Zielgrößenwerten für jedes Experiment wurden der Mittelwert, das Minimum und das Maximum bestimmt und eine trianguläre Zugehörigkeitsfunktion gebildet (Abb. 10 links). Da bei dieser Anwendung besonders die Bereiche mit kurzen Antwortzeiten (d.h. kleinen Werten) von Interesse sind, wurde die Granularisierung für kleinere Werte feiner gewählt. (Abb. 10 Mitte und rechts).
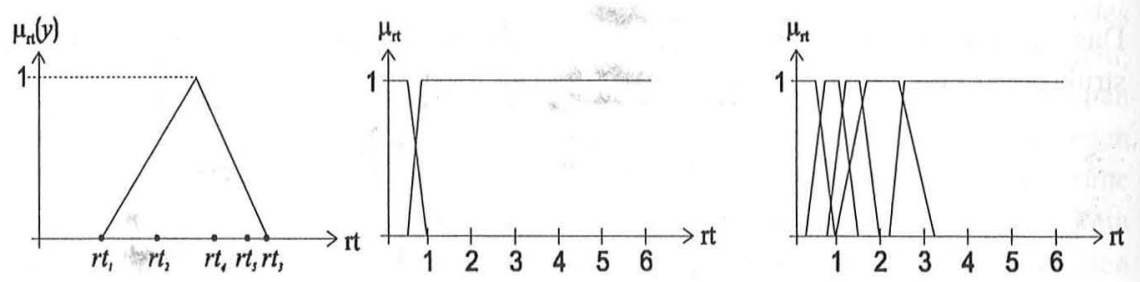

Abb. 10: Trianguläre Zugehörigkeitsfunktionen eines Zielgrößenwertes (links). Zwei Granularisierungen der Zielgröße (Mitte und rechts).

Die Generierung des Fuzzy Graphen erfordert keine weiteren Parametrisierungen und benötigt für dieses Modell mit der aktuellen Implementierung auf einer Sun Sparc 10 Workstation etwa 10 Sekunden. Die Approximation von Zielgrößenwerten erfolgt sogar in Sekundenbruchteilen, während ein Simulationsexperiment zur Bestimmung eines Zielgrößenwertes etwa 200 Sekunden benötigt. Damit ist das Metamodell um mindestens zwei Größenordnungen schneller bei der Approximation neuer Eingabeparameter, d.h. es kann für die Durchführung neuer Experimente als „Simulator" genutzt werden.

Um die Glaubwürdigkeit des generierten Metamodells zu überprüfen, kann der mittlere relative Approximationsfehler betrachtet werden. Dazu wurde ein 10faches Crossvalidation durchgeführt. Der komplette Datensatz mit 350 Beispieldaten wurde aufgeteilt in 9/10 Trainings- (315) und 1/10 Testdaten (35). Mit den Trainingsdaten wurde der Fuzzy Graph aufgebaut, und für alle Testdaten wurde der approximierte Wert durch das Metamodell mit dem bekannten Wert des Testdatums verglichen und die mittlere relative Abweichung berechnet. Dies wurde insgesamt zehnmal durchgeführt mit jeweils unterschiedlichen Aufteilungen wows 
der Trainings- und Testdaten. Die jeweiligen mittleren Fehler wurden über 10 Trainings/Testzyklen gemittelt und sind in Tabelle 1 eingetragen:

Tab.1: Approximation des Fuzzy Graph Metamodells

\begin{tabular}{|c|c|c|c|}
\hline & 2 Klassen & 5 Klassen & 10 Klassen \\
\hline $\begin{array}{c}\text { Mittlere relative } \\
\text { Abweichung auf den } \\
\text { Testdaten }\end{array}$ & $4.4 \%( \pm 1.0 \%)$ & $4.1 \%( \pm 1.2 \%)$ & $3.3 . \%( \pm 0.9 \%)$ \\
\hline $\begin{array}{c}\text { Mittlere Anzahl an } \\
\text { erzeugten Regeln }\end{array}$ & 32 & 59 & 68
\end{tabular}

Durch eine Erhöhung der Klassen wird der Fehler erwartungsgemäß kleiner und die Regelanzahl steigt an. Ein deutlich kleinerer Fehler kann kaum erwartet werden, da der Fuzzy Graph durch die Generalisierung Abweichungen im gewissen $\mathrm{Maß}$ toleriert. Im Kontext der Simulation ist diese Robustheit durchaus erwünscht, zumal die Zielgrößenwerte beim Training gerade die Unschärfe beinhalten. Außerdem ist das Ziel, in erster Linie ein verständliches Metamodell zu generieren und nicht extrem kleine Abweichungen zwischen Modell und Metamodell zu erreichen.

Bei der Anwendung von Metamodellen ist nicht nur der mittlere (oder maximale) Approximationsfehler auf Testdaten von Bedeutung, sondern auch ob das Verhalten des Metamodells dem grundsätzlichen Verhalten des Modells entspricht. Da eine Visualisierung des Verhaltens nur bei zwei Eingabedimensionen möglich ist, wurden die zwei Eingabeparameter „Antwortzeit der CPU“ und die „Wartezeit zwischen Aufträgen“ ausgewählt und der entsprechende Zielgrößenwert aufgetragen (Abb. 11).
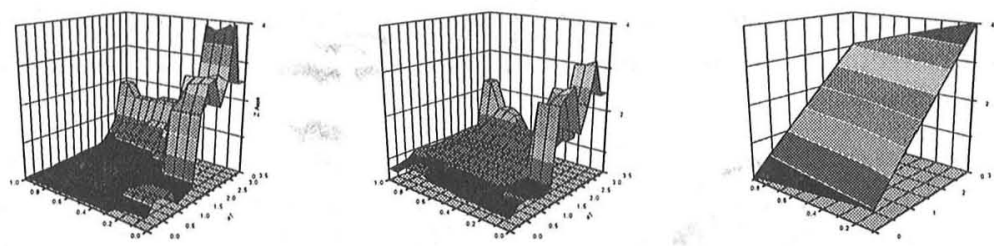

Abb. 11: Modellverhalten, Fuzzy Graph und lineare Regression

Man erkennt am Verhalten des Modells (Abb. 11 links), daß die Modellfunktion hochgradig nichtlinear ist. Es gibt einen weiten Bereich, in dem die Zielgrößenwerte sehr niedrig sind, und die Werte steigen stark an, wenn die „Antwortzeit der CPU“ sich dem oberen Grenzwert nähert, insbesondere, wenn die „Wartezeit" geringer wird. Dies entspricht intuitiv der Erwartung an das Modellverhalten, denn bei einer langsamen CPU (hohe Antwortzeiten) und einer hohen Belastung (geringe Wartezeit zwischen Aufträgen) würde man auch große Antwortzeiten erwarten. Andererseits ist es wichtig zu wissen, in welchen Bereichen sich die Zielgröße ,gutmütig“ verhält, bzw. wo ein Anstieg der Antwortzeiten erfolgt. Daher 
muß ein Metamodell diese Bereiche hinreichend gut wiedergeben. Das Verhalten des Metamodells (Abb. 11 Mitte) zeigt eine große Übereinstimmung mit dem des Modells. Sowohl die Regionen, in denen sich die Zielgröße wenig ändert, als auch die Bereiche mit starken Schwankungen werden sehr gut nachgebildet. Das bedeutet, daß das Fuzzy Graph Metamodell trotz der sehr kompakten Struktur auch komplexe Modellfunktionen nachbilden kann.

Zum Vergleich wurde für die hier dargestellte zweidimensionale Modellfunktion auch eine lineare Regressionsgleichung bestimmt (Abb. 11 rechts). Die Regressionsfunktion gibt zwar die grundlegende Richtung der Zielgrößenwerte bezüglich der Eingabeparameter an, aber nur sehr grob. Durch die Wahl einer geeigneteren nichtlinearen Regressionsfunktion kann die Übereinstimmung zur Modellfunktion verbessert werden ${ }^{1}$, dennoch ist es sehr unwahrscheinlich, daß die einzelnen Regionen des Modellverhaltens explizit erkennbar werden. Dies ist u.a. darin begründet, daß der Regressionsansatz eine globale Beschreibung des Metamodells in Form der Regressionsgleichung erzeugt. Der Fuzzy Graph ist dagegen aufgrund der individuell generierten Fuzzy Punkte ein lokaler Ansatz, dem dadurch meist eine genauere Anpassung an die Modelldaten und damit an die Modellfunktion gelingt.

Durch die gute Nachbildung des Modellverhaltens können Simulationsexperimente eingespart werden, indem das Metamodell zur Bestimmung neuer Zielgrößenwerte eingesetzt wird, beispielsweise zur Optimierung der Modellparameter bezüglich einer minimalen Antwortzeit. Da zudem die relevanten Regionen gut nachgebildet wurden, repräsentieren auch die Regeln des Metamodells das Modellverhalten hinreichend genau und können zur Analyse des Modellverhaltens genutzt werden.

Der Approximationsfehler ist bereits bei zwei Zielgrößenklassen sehr gering, so daß im folgenden dieses Metamodell näher untersucht wird. Zur besseren Verständlichkeit sind die beiden Klassen mit „low“ und „high“ bezeichnet. Von den 32 erzeugten Regeln gehören 17 Regeln zur Klasse low und die Regel mit dem größten Gewicht lautet:

$\begin{array}{llllll}\text { Wenn } & \text { CPU_Antwortzeit } & \text { in } & {[0.11,1.69]} & \subset & (\mathrm{ub}, 1.70) \\ \text { und } & \text { Wartezeit } & \text { in } & {[0.03,0.99]} & \subset & (\mathrm{ub}, \mathrm{ub}) \\ \text { und } & \text { Target-Rotation-Time } & \text { in } & {[0.01,0.39]} & \subset & (\mathrm{ub}, \mathrm{ub}) \\ \text { und } & \text { Anzahl_Stationen } & \text { in } & {[5,15]} & \subset & (4, \mathrm{ub}) \\ \text { Dann } & \text { Mittlere_Antwortzeit ist low } & \text { in } & {[0.0,0.5]} & \subset & (\mathrm{ub}, 1.0) \\ \text { Regelgewicht: } 116 & & & & \end{array}$

Diese Regel zeigt, wie der Kern immer einen Teilbereich des Einflußbereichs überdeckt. Der Kern überdeckt bei den zwei Parametern Wartezeit und TargetRotation-Time den kompletten Wertebereich. Die beiden anderen Parameter sind nur im Einflußbereich in jeweils einer Richtung beschränkt ( $u b=$ unbeschränkt).

\footnotetext{
${ }^{1}$ Sofern sich eine solche Funktion in der Praxis überhaupt finden und dann effizient berechnen läßt.
} 
Die Antwortzeit sollte unter 1.70 Zeiteinheiten liegen und die Anzahl zusätzlicher Stationen sollte größer als 4 sein. Daraus läßt sich ablesen, daß bei einer hinreichend schnellen CPU und einer Hintergrundlast kurze Antwortzeiten erreicht sind, unabhängig davon, wie der Netzparameter trt eingestellt wird und wie hoch die Anzahl der Anfragen pro Zeitintervall ist. Das Gewicht dieser Regel entspricht der Anzahl von Beispieldaten, die vom Kern dieser Regel überdeckt werden, d.h. diese Regel hat eine hohe Glaubwürdigkeit. Regeln mit kleinen Gewichten können dagegen Hinweise auf Ausreißer oder Unregelmäßigkeiten in den Daten, sowie auf Regionen mit hoher Empfindlichkeit liefern.

Eine weitere interessante Fragestellung ist der Einfluß einzelner Parameter auf die Werte der Zielgröße. Es läßt sich beispielsweise aus obiger Regel schließen, daß bei einer genügend schnellen CPU der Parameter Target-Rotation-Time kaum Einfluß hat. Andere Regeln zeigen, daß bei anderen Einstellungen die TargetRotation-Time größer als 0.25 sein muß, um kurze Antwortzeiten zu erreichen. Hier beeinflußt dieser Parameter offensichtlich die Zielgröße. Neben der Untersuchung der Regionen, in denen kurze Antwortzeiten erreicht werden, kann auch die Betrachtung der anderen Regionen (also von Regeln der Klasse high) sinnvoll sein. Dies gibt zum Beispiel Hinweise auf Parametereinstellungen oder auf Lastprofile, die im praktischen Einsatz vermieden werden sollten.

Die Regeln des Metamodells liefern somit verständliche Hinweise auf Abhängigkeiten zwischen Modellparametern und der betrachteten Zielgröße. Durch die Überprüfung des Approximationsfehlers kann einerseits abgeschätzt werden, in welchem Bereich die Werte schwanken können, und andererseits ist eine Beurteilung der Qualität des Metamodells möglich. Wird hohe Approximationsgenauigkeit gefordert, müssen entsprechend viele Simulationsexperimente durchgeführt werden. Zusätzlich kann die Granularisierung mit mehr Klassen erfolgen, was aber $\mathrm{zu}$ einer feineren Aufteilung des Eingaberaumes und $\mathrm{zu}$ schlechterer Interpretierbarkeit führen kann.

\section{Zusammenfassumg}

Die Verhaltensanalyse komplexer Systeme wird in der Praxis häufig an Modellen durchgeführt, für die wiederum leistungsfähige Analysemethoden benötigt werden. Ein Weg besteht darin, die Modelle auszuwerten oder zu simulieren und aus den gewonnenen Daten ein verhaltensgleiches Metamodell zu generieren. Dazu wurde ein neuer Ansatz vorgestellt, der aus den Daten automatisch einen Fuzzy Graphen generiert. Dieses Konzept erlaubt die Approximation auch komplexer Modellfunktionen und ermöglicht auch, stochastisch verrauschte Informationen zu verarbeiten. Am Beispiel eines komplexen Simulationssystems wurde gezeigt, daß sich das erzeugte Fuzzy Graph Metamodell dazu eignet, das zugrundeliegende Modellverhalten nachzubilden. Die Fuzzy Punkte können als lokale Wenn-Dann-Regel dargestellt werden, die vom Modellierer direkt analysiert und interpretiert werden können. Sie geben Hinweise u.a. auf Modelleinstellungen und auf Einflüsse der 
einzelnen Eingabeparameter, und sie sind durch die einfache Lesbarkeit eine sehr verständliche Repräsentation des Modellverhaltens.

\section{Literatur}

Abe, Shigeo und Ming-Shong Lan 1995: Fuzzy Rules Extraction Directly from Numerical Data for Function Approximation; IEEE Transactions on Systems, Man, and Cybernetics, Vol. 25, No. 1, january 1995, pp. 119-129.

Bandemer, Hans und Siegfried Gottwald 1993: Einführung in Fuzzy Methoden Theorie und Anwendungen unscharfer Mengen; Akademie Verlag, Berlin, 1993

Higgins, Charles M. und Rodney M. Goodman 1993: Learning Fuzzy Rule-based Neural Networks for Control; Advances in Neural Information Processing Systems, NIPS, 5, California, Morgan Kaufman, S. 350-357.

Huber, Klaus-Peter 1997: Fuzzy Graph Metamodelle - Datenbasierte Verhaltensanalyse komplexer Systeme; Dissertation an der Fakultät für Informatik, Universität Karlsruhe, in Vorbereitung.

Huber, K.-P. und Michael R. Berthold 1995: Building Precise Classifiers with Automatic Rule Extraction; Proceedings of the IEEE International Conference on Neural Networks, vol.3, Perth, S. 1263-1268.

Hurrion, R. D. 1992: Using a Neural Network to Enhance the Decision Making Quality of a Visual Interactive Simulation Model; Journal of the Operational Research Society, Vol. 43, No. 4, S. 333-341.

Kleijnen, Jack P. C. 1979: Regression Metamodels for Generalizing Simulation Results; IEEE Transactions on Systems, Man, and Cybernetics, Vol. SMC-9, No. 2, feb., S. 93-96.

Kreimer, Joseph 1992: Simulation and Sensititvity Analysis of Discrete Event Systems; Proceedings of the 1992 European Simulation Multiconference, York, ESM 92, S. 167-171.

Pierreval, Henri 1992: Rule-based Simulation Metamodels; European Journal of Operational Research, 61, Elsevier Science Publishers B.V., S. 6-17.

Pierreval, Henri 1996: A Metamodelling Approach Based on Neural Networks; International Journal of Computer Simulation, 6(2).

Syrjakow, Michael 1997: Verfahren zur effizienten Parameteroptimierung von Simulationsmodellen; Dissertation an der Fakultät für Informatik, Universität Karlsruhe.

Wang, Li-Xin und Jerry M. Mendel 1992: Generating Fuzzy Rules by Learning from Examples; IEEE Transactions on Systems, Man, and Cybernetics, Vol. 22, No. 6, Nov/Dec, S. 1414-1427.

Zadeh, Lotfi A. 1994: Fuzzy Logic, Neural Networks, and Soft Computing; Communications of the ACM, Vol. 37, No. 3, march, S. 77-84. 